\title{
OPTIMIZATION OF OIL FIELD DEVELOPMENT PROCESS BASED ON EXISTING FORECAST MODEL
}

\author{
Vladimir Lushpeev ${ }^{1 *}$, Andrey Margarit ${ }^{2}$ \\ ${ }^{1}$ Saint Petersburg State University, Saint Petersburg, Russian Federation \\ ${ }^{2}$ Gazpromneft NTC LLC, Saint Petersburg, Russian Federation
}

Process of oil-and-gas field development optimization under the conditions of a mineral raw material base deterioration and increase in a share of hard-to-recover reserves is the integral part of commercial production stage, especially in the last stage of development. Decisions regarding the optimization of the development system with contour water flooding under the conditions of a high water-cut of well production need to be made using additional instruments for the decision making, such as 1-D, 2-D and 3-D models. Using of simulation does not exclude a participation of experts in such work and imposes great responsibility on them in making decisions. Searching for optimal decisions under the oil-and-gas field development optimization based on physic-mathematical models together with the participation of recovery and development experts is the basis for managerial decision making in oil-and-gas production companies. This article shows the principles of the oil-and-gas field development optimization based on the existing forecast model and describes an industrial example of such optimization instrument usage together with the participation of the experts.

Key words: Oils, Optimizer, Development processes, Oil field

\section{INTRODUCTION}

The results of simulating a development optimization project are only the implementation of certain conditions with some degree of approximation. Therefore, the model has an error, a risk and restrictions and does not take into account all effects, but only those that can be described with a selected degree of detail $[9,10]$.

In this connection the restrictions unaccounted in the simulation can be arose at the different stage of the project implementation, for example, an operating wells equipment capabilities, probable non-predicted effects under a complex configuration of a real reservoir or the development system.

To successfully solve the problems regarding the oil field management the organization of multidisciplinary teams involving the experts of different branches had been effectively realized in many oil-and-gas companies. However, such approach requires the expenditures of a significant quantity of labour resources and organizational capacities that can be ineffective.

$$
f(\mathrm{x})=\mathrm{NPV}(\mathrm{x})=\sum_{k}\left[\sum_{j} \mathrm{p}_{o} q_{o}^{j . k}(\mathrm{x})-\sum_{j} \mathrm{c}_{l p} q_{l p}^{j . k}(\mathrm{x})-\sum_{j} \mathrm{c}_{w i} q_{w i}^{j . k}(\mathrm{x})\right] \Delta t_{k} /(1+d)^{t_{k}}
$$

Where $p_{0}, c_{i p}, c_{w i}$ - the oil cost (which already includes costs for separation and tax levies), lifting costs and costs for water injection, accordingly, $q_{0}^{j \cdot k} q_{10}^{j \cdot k} q_{w i}{ }^{j k}-$ oil and fluid production rates, water injection rate for each well $j$ at every time interval $k$ in the time period $\Delta t_{k}, d-$ yearly discount rate.

A boundary conditions was also imposed, i.e. $\mathbf{x}$ was defined as $x=\left\{x \in R^{n} ; x_{d} \leq x \leq x_{u}\right\}$, where $x_{d}$ and $x_{u}$ designate the lower and upper bounds accordingly. In addition to these boundary conditions the nonlinear restrictions were taken into account, which were mathematically described
As one of the methods to overcome such restrictions may be the expertise of the optimization project by production experts on site. It also seems appropriate to always adhere to a principle of preliminary testing of the proposed modifications at separate areas of the existing site within the framework of pilot production.

A similar approach to the implementation of the development optimization project was studied on the example of one of the oil fields in Western Siberia.

\section{THEORY AND EXPERIMENTAL METHODS}

In order to set the optimization task the variables optimization method $x \in R^{n}$, represented by the variety of test parameters of the well performance in certain time intervals, was chosen. The bottom-hole pressure (BHP) was used as the $\mathbf{x}$ variable for this task. A net present value (NPV) was chosen as an optimizable function of cost $f(x)$ for relatively short time interval (one to three years):

by vector function $k(x) \in R^{n k}$, whose components have to be nonpositive for possibility of decision. These components in the task to solve depend on the value of recovery efficiency (RE) estimated for the same time interval as for the NPV (this RE should be greater than the average value for development). As a result the optimization task can be stated as follows:

$$
\mathbf{X}^{*} \in \arg \max _{x \in \mathrm{X}} f(\mathbf{x}) \text {, provided that } \mathbf{k}(\mathbf{x}) \leq 0 \mathbf{x} \in \mathbf{X}
$$

where $\mathbf{X}^{\star}$ designates any certain (local) optimal decision, 
the one of the variety, and the exact value of nonlinear restriction is adjusted so that it is nonpositive [8].

\section{OPTIMIZATION METHODS}

In view of the above stated conditions, it was decided to apply the optimization methods without using of derivatives (derivative-free). Most of performed experiments are based on Generalized Pattern Search technique, GPS $[1,3,7]$. As mentioned above, in the task of development control, as a rule, there are multiple optimums with the similar values of cost function that is used in this technique. Besides, the universal procedure Particle Swarm Optimization, PSO was tested on the one of the examples $[2,5,6]$. In both algorithms the analysis of the searching area was performed using a set of points (a pattern for GPS and a variety for PSO), which were defined for each iteration.

It is worth pointing out that time costs for calculations by methods without using the derivatives depend on quantity of optimizable variables. Therefore, the optimization of the oil fields at the late stage of development with relatively large quantity of wells is time-consuming.

The applied method assumes the searching of the optimal value $\mathrm{q}_{\mathrm{wi}}{ }^{\mathrm{k}} \cdot \mathrm{x}$ ), i.e. the injection capacity of well (that restricts the number of variables), which will correspond to maximum NPV value. Then the searching results shall be tested on heological-hydrodynamical model (HHDM) of the oil field.

\section{THE OIL FIELD DESCRIPTION}

The optimization method was tested on the one of the oil fields of PJSC "Gazprom neft», located in Western Siberia. The deposit is the layer-uplifted deposit with elements of tectonic shielding. Reservoir is generally represented by sandy rock with beds of siltstone, hard clays and carbonate rocks, which divide the formation into 9-28 permeable partings. The reservoir's plan dimensions are $12 \times 4.3 \mathrm{~km}$, the total thickness is $51 \mathrm{~m}$. The average thickness of clean sand is 25 meters, the initial oil-filled thickness is from $1.1 \mathrm{~m}$ to $25.6 \mathrm{~m}$. The average porosity for the field is $18.8 \%$, the average permeability $23.6 \mathrm{mD}$, the average initial oil saturation $-61 \%$.

Exploratory drilling confirmed the oil reserves in 1989 , the development was begun in 2006. Oil recovery obtained on vertical exploration wells amounted 723 barrels per day, and on horizontal producing wells $-3,189$ barrels per day. Maximum oil recovery was about 17,000 barrels per day from 2007 to 2012 . Water flooding process happens effectively and without sharp breachings of displacement front as evidenced by a gradual increase in water-cut (see Figure 1).

Before the optimization of water flooding in May, 2016 the oil field was on the fourth development stage with the oil production rate of 6,440 barrels per day (see Figure 1). The stock has 19 production wells (including 16 horizontal wells) and 15 injection wells (one of them is horizontal well).

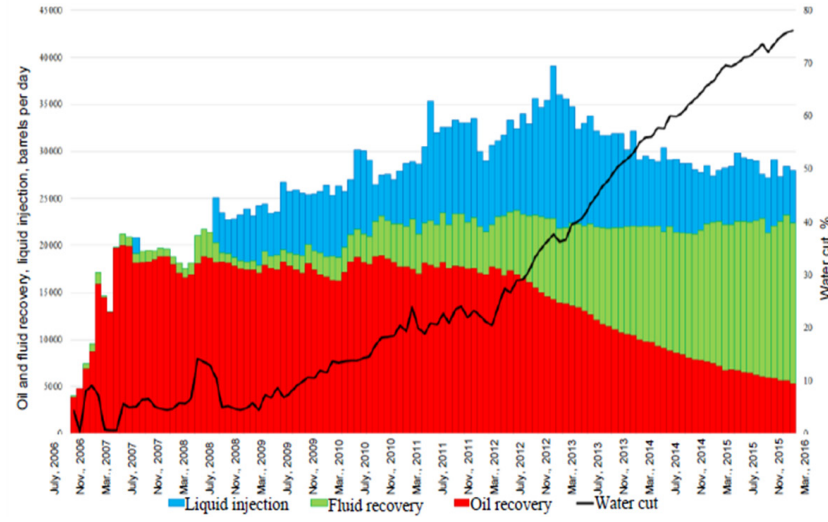

Figure 1: recovery profiles for the examined oil field

The maps of the development and the oil-filled thicknesses as of April 30, 2016 are shown on Figure 2.

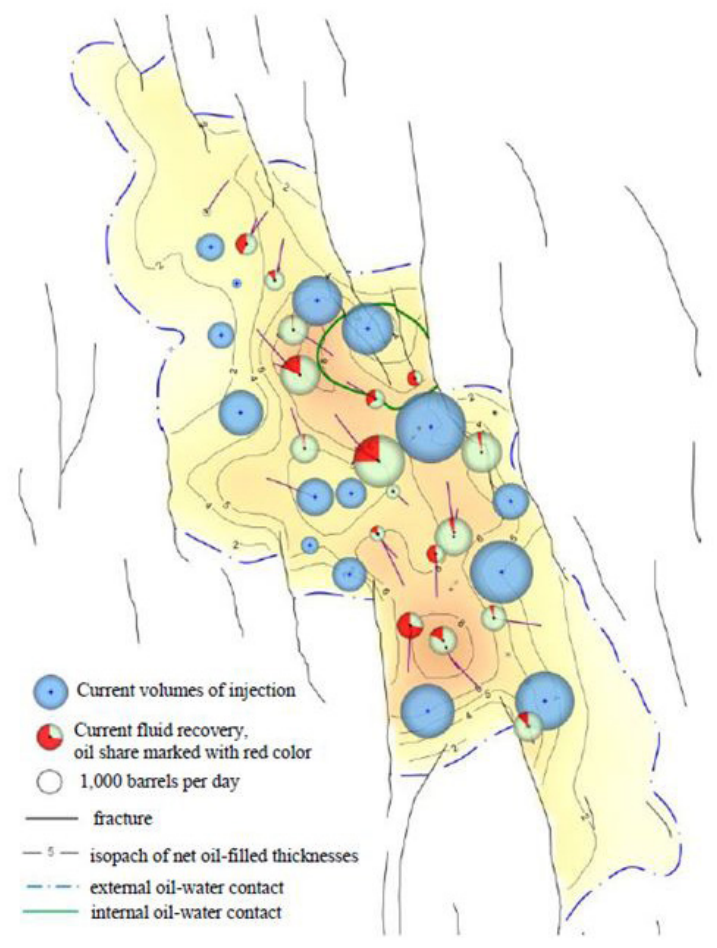

Figure 2: The maps of the development and oil-filled thicknesses, 30.04.2016

For timely monitoring and optimization the oil field's HHDM was used. It contains $70 \times 145 \times 114$ grid blocks (768,000 of them are active) with horizontal dimension of the grid block of $100 \times 100$ meters and the average thickness of 0.3 meter. The quality of the model history match is considered satisfactory for measures to optimize and monitor of the effect.

\section{DEVELOPMENT OPTIMIZATION PROJECT ON HYDRODYNAMIC MODEL}

As a result of solving the water flooding optimization problem, three injection wells were identified for possible changes of the injection mode (see Figure 3). Main recommendation relates to the injection well INJ-2: optimal decision is achieved particularly due to the well shutting 
in (i.e. reducing the injection capacity of the well by $100 \%)$. Additionally, it was recommended to increase the injection in INJ-1 and INJ-3 wells. The obtained decision can be considered as physically reasonable. High injection capacity of the INJ-2 well can impede the effective hydrodynamic interaction of the INJ-1 well with the adjacent production wells and, consequently, reduces the oil displacement efficiency. The shutting in of the INJ-2 well together with the increasing the injection of the INJ1 well can redistribute the filtration flows and involve the remaining reserves in the area of the specified wells. It was recommended to increase the injection in the INJ-3 due to the good efficiency of filtration between the INJ-3 well and the nearest production wells. The total injection capacity of the INJ-1, INJ-2 и INJ-3 wells should be reduced by $23 \%$. It was assumed that involving of the remaining reserves along with the savings of the costs for the water injection and the lifting of liquid as a result exceeds losses caused by a decrease in formation pressure.

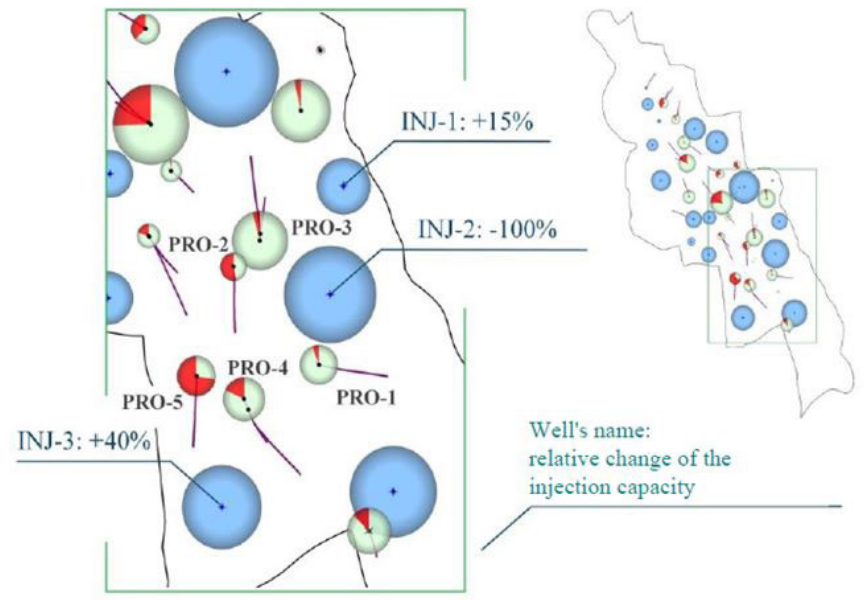

Figure 3: Proposed measures of optimization for water flooding control; the relative changes of injection capacity for each well INJ-1, INJ-2 u INJ-3 are shown.

\section{DECISION MAKING BASED ON SIMULATION RESULTS}

Optimal decision was studied by the experts who are responsible for actual exploitation and the development control of the oil field. Only the injection restraining in the INJ-2 well by $50 \%$ was approved from the proposed optimization measures. The reasons of partial implementation of the proposed measures are the following:

\section{Infrastructure limitations}

There are significant technological limitations for redistribution of the injection using the ground infrastructure. Every well pad has a separate system of water intake and water injection into the formation. The INJ-1 and INJ-2 wells are located at the different well pads; so the redistribution of the injection between them is technically impossible. Note that the injection capacity increase in INJ-1 well without the redistribution of the injection from the other wells is also impossible due to the ultimate capacity of the water intake system in the well pads.

Detailed and timely consideration of such factors becomes possible due to the existence of an integrated model of the oil field. However, the creation, updating and usage of that model for decision making in practice result in significant increase in computational complexity and labour costs. Comparison of an efficiency of the decision making based on the models of various levels of detailing can become the focus for further researches.

\section{High level of uncertainty}

It is obvious that predictable positive effect with high degree of possibility shall to be confirmed in case of its value does not exceed the level of incoming uncertainties. For this certain case there is a high degree of risk (which was not estimated quantitatively) that existing activities will have a negative impact on the development performances:

- when increasing the injection in the INJ-3 well there is high possibility of the breaching of the displacement front to T3 point (bottom hole) of the PRO-5 well;

- a full shut-off of the INJ-2 well which provides about $30 \%$ of the injection at that area of the oil field can lead to more significant decrease of the formation pressure than the predicted one based on HHDM due to the fact that such prolonged shut-offs of the INJ-2 well have not been carried out and, consequently, the model can not be validated for this type of measures.

Therefore, a limited version of the suggested optimization recommendations was applied at the oil field - pilot-industrial works to limit the injection capacity of INJ-2 well by $50 \%$. This allowed making rather small changes in the modes, measuring the dynamics and, depending on the result obtained, expanding or refusing the measures taken. This analysis can be one of the key moments for the estimation of the effect of the full INJ-2 well shut-off. This well has a key role in forming of the water flooding system in the studied oil field region, and therefore, the serious reasons are required for its full shut-off.

\section{RESULTS OF THE OIL FIELD EXPERIMENT IN THE WATER FLOODING CONTROL}

As a result, it was decided to install a flow nipple in the INJ-2 well for a double reduction in the injection capacity.

Figure 4 shows the actual monthly operational reports (MOR) a year before the start of the experiment and for 8 months after. The area with the production wells PRO$1, \ldots-5$ and the injection wells INJ-1, $-2,-3$ is defined as the "area of the experiment» (the region with the highest predictable potential of the optimization). A significant decrease in the injection has been observed from May, 2016 (the start of the experiment), and the recovery stabilization and the water cut reduction are also observed. In order to reasonably estimate the effect of the water 
flooding optimization the parameters of the experiment area and the rest part of the oil field were compared. It is necessary because at the entire oil field, including in the experimental region, the operator continuously performs regular activities for the development monitoring. It is assumed that the effect from these activities is uniformly distributed over all wells of the oil field including the wells of the experiment area. Let's start by considering the effect of decreasing in an injection capacity.
It is shown in Figure 5 that the injection capacity in the experiment area is compared with another one for other wells. Due to the different quantity of the wells in these two groups (3 versus 12), the different scales are used. In order to simplify the effect estimation the total injection rates during the year preceding the experiment (from May, 2015 to April, 2016) were approximated on the mean value to determine a "basis" injection capacity. As it is shown in the Figure 5 , the value of this parameter is 7.090 barrels ner dav.

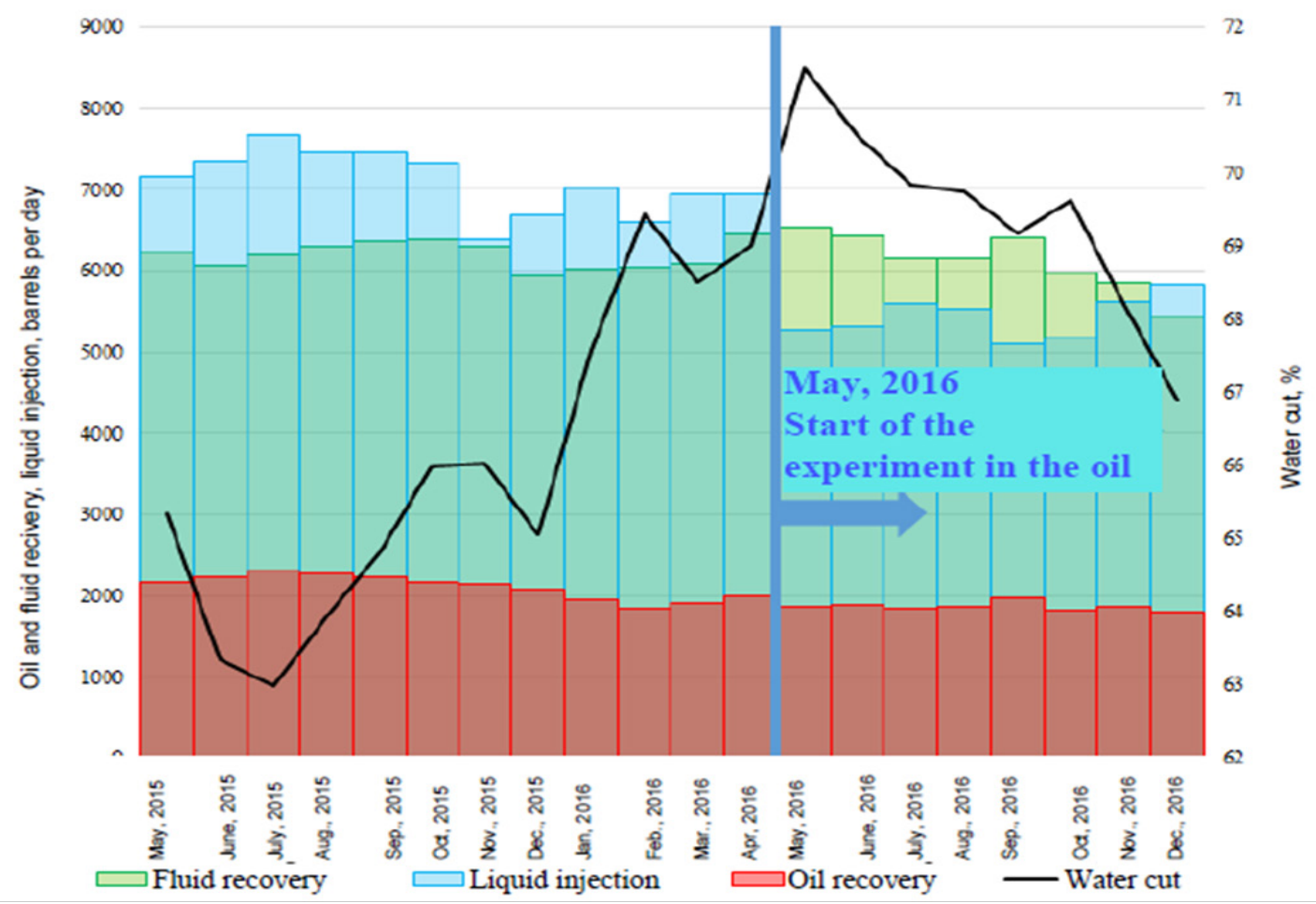

Figure 4: The results of the oil field experiment: a cumulative oil and fluid recovery and water cut of the PRO-1,... -5 and a cumulative injection for INJ-1, INJ-2 and INJ-3 wells. A year before the start of the experiment and for 8 months after.

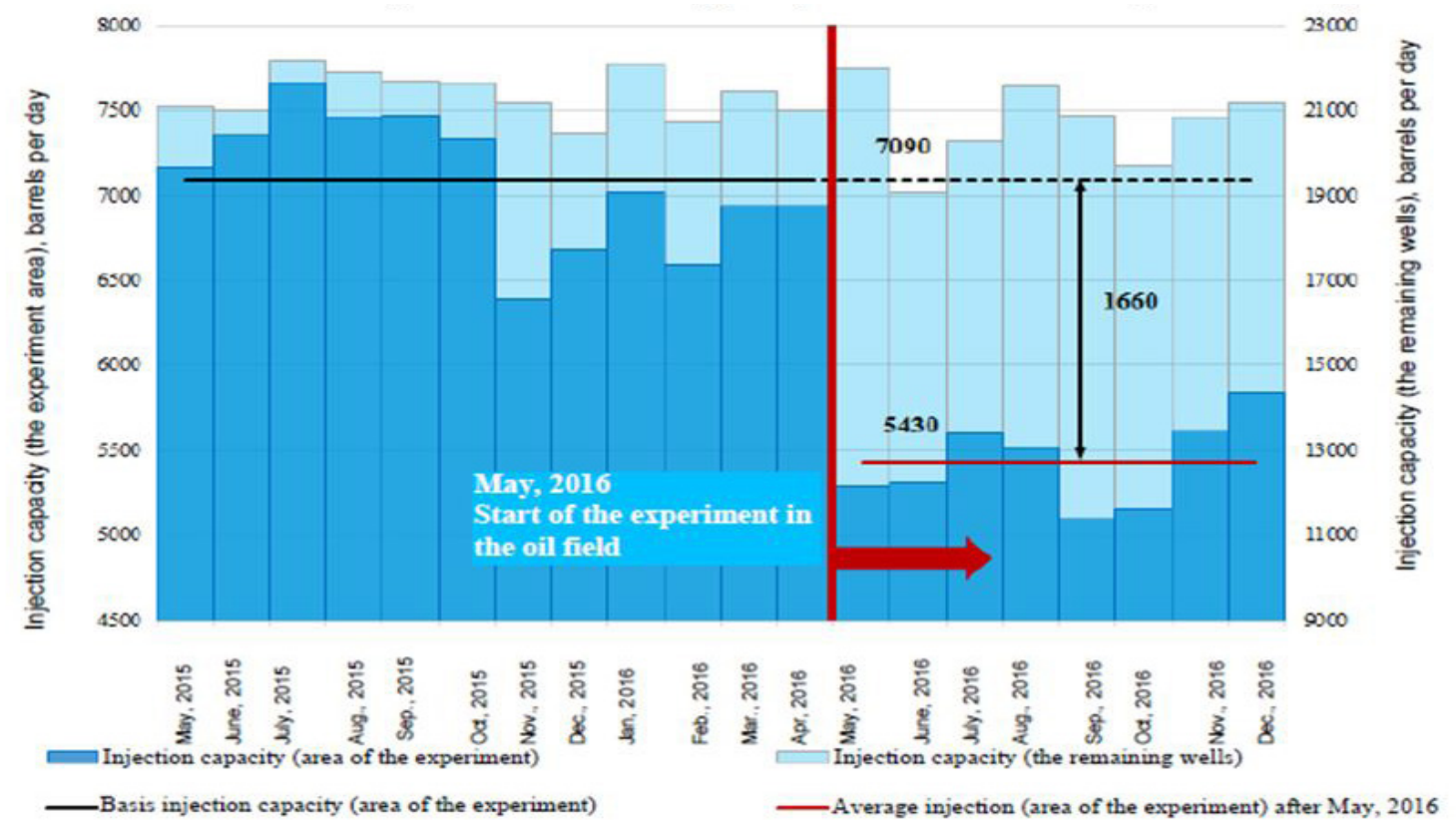

Figure 5: Comparison of total injection from the INJ-1, INJ-2 and INJ-3 wells (the experiment area), with the remaining 12 injection wells 
A variance of the injection liquid volume equal to 390 barrels per day (it is nor shown in the Figure), can be an approximate estimation of the error of the "basis" value. According to a mean reduction of the injection capacity $(1,660$ barrels per day), the effect obtained is in the range of 311,000 to 502,000 barrels within 8 months (245 days) of the experiment. However, this estimation can not be considered as the final result: as stated above, it is required to take into account the operator's continual optimizations over all wells of the oil field. To do this it is required to repeat the similar operation for wells which are not in scope of the experiment region. The decreasing in an injection capacity by "operator" is varied from 40,000 to 304,000 barrels: this effect shall be normalized by equal quantity of wells ( 3 wells) and be deducted from the previous one. Consequently, a "net" effect is in the range from 235,000 to 492,000 barrels and provides the appropriate decreasing of the operational costs.

Figure 6 shows the decrease in dynamics of fluid recovery for both areas of the oil field (a corresponding water cut for each area is also shown in the Figure). If we repeat the above described estimation process we will find that average decreasing of the fluid volume recovered in the experiment area and in the other part of the oil field is 19,000 barrels and 241,000 barrels, accordingly. Fluid rate in the remaining area is vastly larger than the corresponding rate in the experiment area (even if we take into account a normalization coefficient that in this case is taken to be equal to 2.6). In this regard it was estimated that the optimization considered can provide relatively small increase in the fluid production volume and we evaluate this increase to be about 73,700 barrels. Assuming that the water cut in the experiment area decreases somewhat more noticeable than in the remaining part of the oil field, this result can point at higher relative oil recovery for the project optimization with respect to a general approach.

Oil recovery performances for both areas of the oil field are shown in Figure 7. In order to estimate the effect based on the oil recovery, the oil recovery is approximated by linear function which is often a rational approximation for a short terms (as well it is an approximation of exponential decline rate which in the scale of 1 year in this case does not distinguished from the linear). By means of such estimation it was found that average increase in a volume of oil recovery produced relative to the initial level for the experiment area and the rest of the oil field is 25,300 barrels and 24,700 barrels, respectively. If taking into account that the total oil recovery in the second area is about 1.9 times greater than in the first one, then we will obtain the effect approximately equals to 12,000 (it is better to round well in the end) barrels of oil.

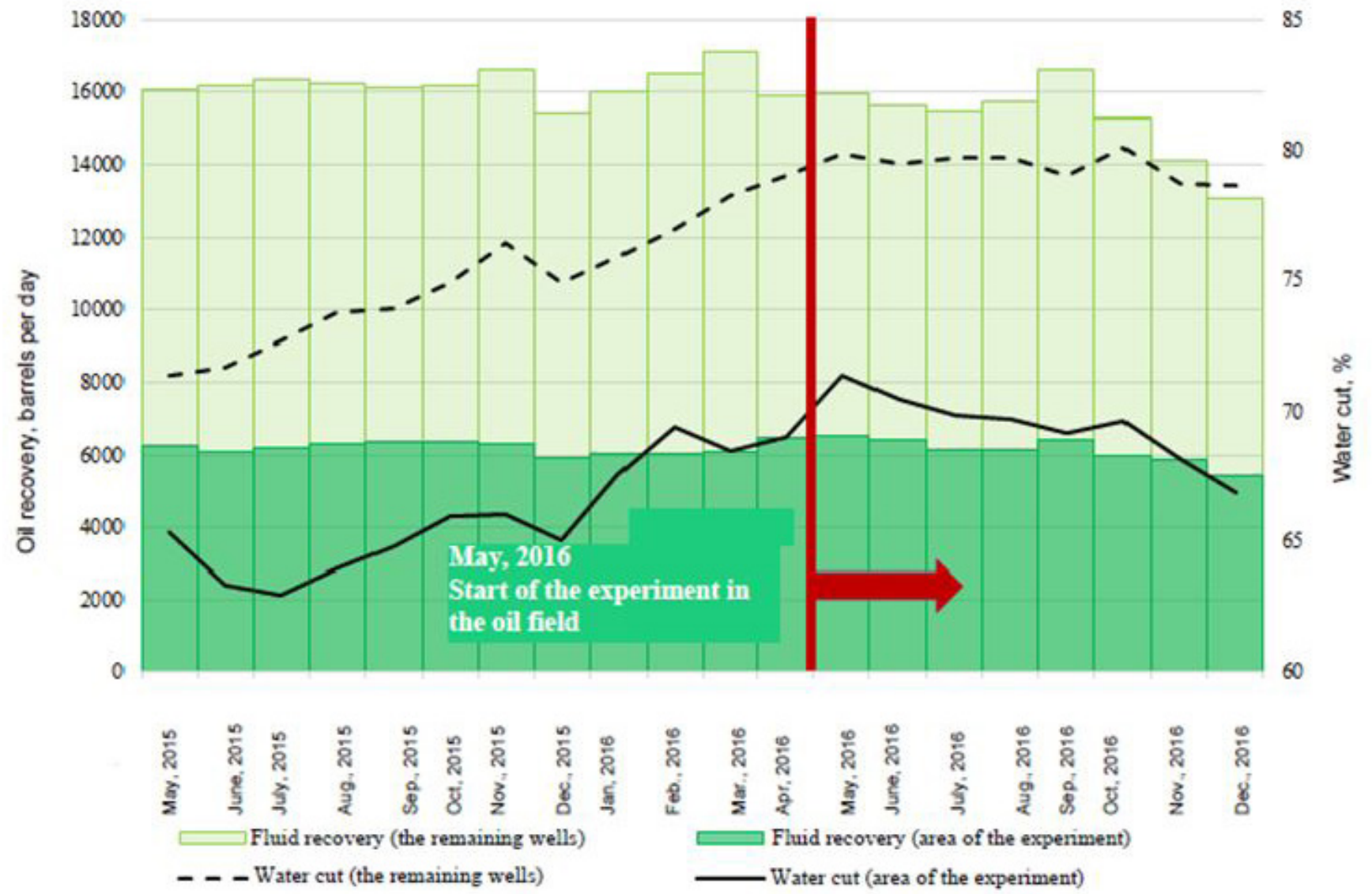

Figure 6: Fluid recovery and water cut over "the experiment area" (PRO-1...-5) and over the remaining 14 development wells of the oil field 


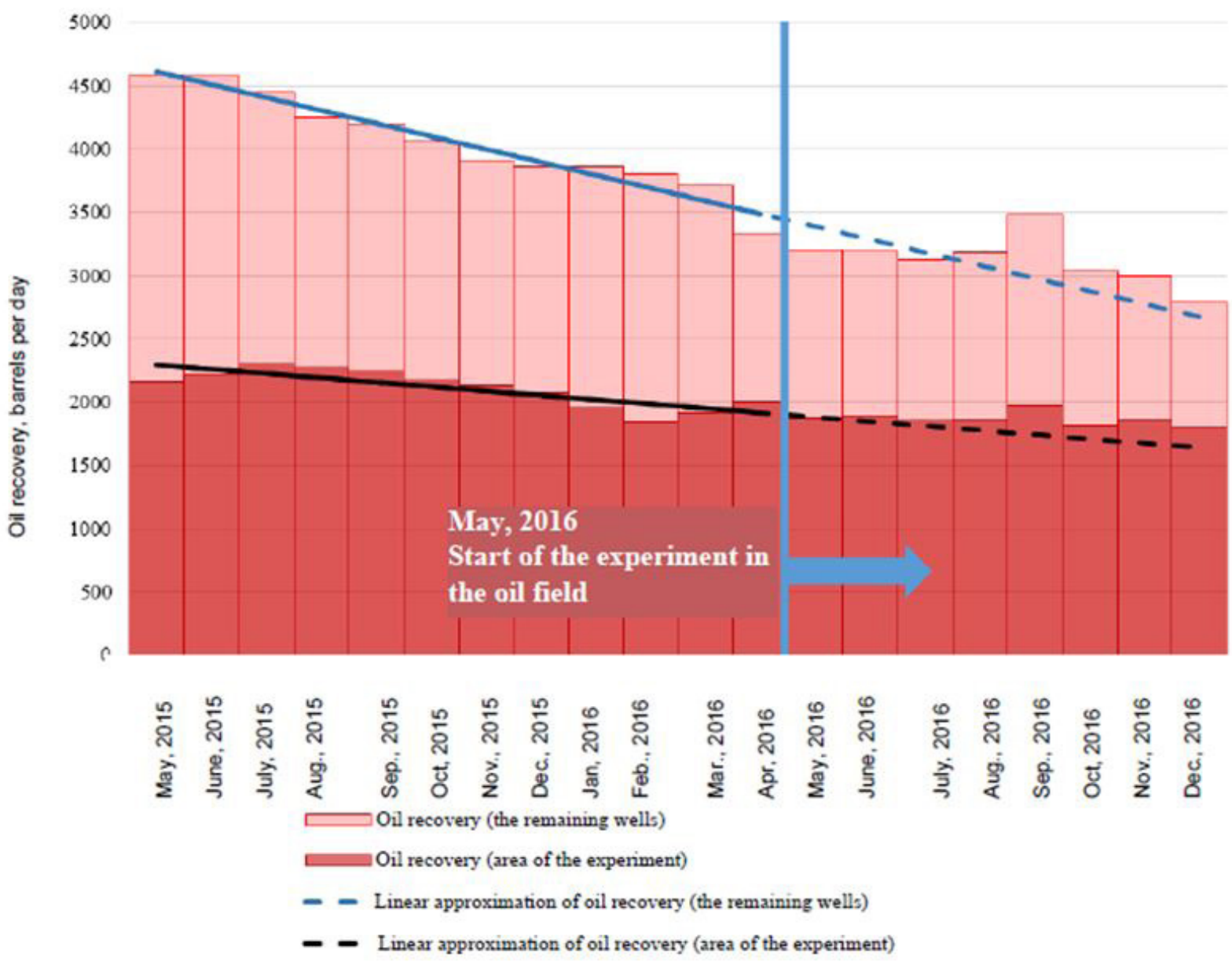

Figure 7: Dynamics of oil recovery for the wells of "the experiment area" (PRO-1,..-5) and for the remaining 14 development wells of the oil field. Linear decline rate which is a good approximation for short period of time is shown for both groups of the wells.

Overall results of the experiment show that the application of the approach presented in this article is economically reasonable. The increase in oil recovery and the decrease in costs for water injection prevail over the slightly higher lifting costs.

\section{CONCLUSIONS}

This article presents an example of optimization of the oil field development, estimated on the basis of the existing hydrodynamic model, and the stages of its implementation and results of the experiment are shown.

Under the existing conditions of the project implementation, the importance of the expert assessment by the specialists in the oil field, which are responsible for management of the field development, is presented. During the implementation of the optimization project they performed the risk analysis that led to adjustment of scheduled. It is probably that in case of more complicated integrated model and a qualitative uncertainty analysis, it would be possible to take this into account beforehand, but creating such a model requires much more organizational and time resources [4].
The results of the water flooding optimization experiment were recognized as positive, however, the analysis of the results showed that the model does not have a high predictive power, and it was decided to refuse the full shutting in of the INJ-2 well, leaving the current mode. Besides, this experiment is a good example of initiating a cyclic working process based on the optimization of the development in a mature oil field: significant changes in the operating modes of the wells, which may be contrary to the usual approach to planning, lead to useful responses in the dynamics of the field factors. These results can be used in the updating and validation of HHDM and the transition to the next optimization iteration.

\section{REFERENCES}

1. Audet, C. and Dennis Jr., J.E. (2002) Analysis of generalized pattern searches. SIAM Journal on Optimization, 13(3), 889-903.

2. Eberhart, R.C. and Kennedy, J. (1995) A new optimizer using particle swarm theory. Proceedings of the Sixth International Symposium on Micromachine and Human Science, 39-43. 
3. Echeverría Ciaurri, D., Isebor, O.J. and Durlofsky, L.J. (2011) Application of derivative-free methodologies to generally constrained oil production optimization problems. International Journal of Mathematical Modelling and Numerical Optimisation, 2(2), 134-161.

4. Integrated Modelling Approach as Estimation Tool for Well Regimes and Gathering Network Impact on Oil Rim Development (Russian), 182007-RU SPE Conference Paper - 2016.

5. Kennedy, J. and Eberhart, R.C. (1995) Particle swarm optimization. Proceedings of IEEE International Joint Conference on Neural Networks, 19421948.
6. Onwunalu, J. and Durlofsky, L.J. (2010) Application of a particle swarm optimization algorithm for determining optimum well location and type. Computational Geosciences, 14, 183-198.

7. Torczon, V. (1997) On the convergence of pattern search algorithms. SIAM Journal on Optimization, 7(1), 1-25.

8. Van Essen, G., Van den Hof, P.M.J. and Jansen, J.D. (2011) Hierarchical long-term and short-term production optimization.109 SPE Journal, 16(1), 191-199.

9. Lushpeev V.A., Tsiku Y.K., Sorokin P.M. (2014) Welltest during synchronous-separate operation. Life Science Journal 2014; 11(12s):351-353] (ISSN:10978135). http://www.lifesciencesite.com. 73

10. J.R. Fanchi, (2000). Integrated approach. ANCO «Institute of computer researches». 